\title{
Prognostic factors of in-hospital mortality in all comers with ST elevation myocardial infarction undergoing primary percutaneous coronary intervention
}

\author{
Songsak Kiatchoosakun, Chaiyasith Wongwipaporn, Burabha Pussadhamma
}

Division of Cardiology, Department of Medicine, Faculty of Medicine and Queen Sirikit Heart Center of the Northeast, Khon Kaen University, Khon Kaen, Thailand

\section{Correspondence to} Dr Songsak Kiatchoosakun, Division of Cardiology, Department of Medicine, Khon Kaen University, Khon Kaen 40002, Thailand; sonkia@kku.ac.th

Received 8 December 2015 Revised 26 May 2016 Accepted 27 May 2016

\begin{abstract}
Background The prognostic factors of in-hospital mortality in all comers and unselected patients with ST elevation myocardial infarction (STEMI) undergoing primary percutaneous coronary intervention (PCI) have not been well established.

Objective To identify the predictive factors of inhospital mortality in patients with STEMI undergoing primary $\mathrm{PCl}$ in a tertiary heart centre.

Methods Between January 2008 and December 2011, all patients with STEMI undergoing primary $\mathrm{PCI}$ were retrospectively included in this study. Baseline characteristics and angiographic data were reviewed and recorded. The study endpoint was all-cause in-hospital mortality.
\end{abstract}

Results Of the 541 patients included in the study, 63 (11.6\%) died during hospitalisation. Cardiogenic shock at admission was recorded in 301 patients (55.6\%) and 424 patients $(78 \%)$ had multivessel disease. Median door-to-device time was $65 \mathrm{~min}$. After adjustment for baseline variables, the factors associated with in-hospital mortality included age $>60$ years $(\mathrm{OR} 2.98,95 \% \mathrm{Cl}$ 1.17 to $7.05 ; p=0.01$ ), left ventricular ejection fraction $<40 \%$ (OR 2.53, 95\% Cl 1.20 to 5.36; $p=0.02$ ), and final TIMI flow grade 0/1 (OR 20.55, $95 \% \mathrm{Cl} 3.49$ to 120.94; $p=0.001$ ).

Conclusions Age, left ventricular function and final TIMI flow are significant predictors of adverse outcomes in unselected patients with STEMI undergoing primary $\mathrm{PCl}$.

\section{INTRODUCTION}

Primary percutaneous coronary intervention (PCI) is the reperfusion therapy of choice in patients with acute ST elevation myocardial infarction (STEMI), provided it can be performed in a PCI-capable hospital within the recommended time lines. ${ }^{1-3}$ Improvements in the PCI technique and adjunctive pharmacological therapy have led to lower mortality when compared to thrombolytic therapy alone. ${ }^{45}$ The predictors of mortality, either shortor long-term, have been reported; however, most clinical trials of primary PCI excluded STEMI patients at high risk from cardiogenic shock and heart failure. ${ }^{6-8}$ Furthermore most of those studies were performed in areas where the availability of a catheterisation laboratory was not an obstacle to treatment. The objective of this study was to identify the predictors of in-hospital mortality in unselected patients with STEMI in a tertiary heart centre, because the results might differ from previous studies.

\section{PATIENTS AND METHODS}

This was a retrospective study performed at a single tertiary heart centre in Northeast Thailand. The data included all STEMI patients who underwent primary PCI within 12 hours of symptom onset between January 2008 and December 2011. Patients who underwent rescue PCI and a pharmaco-invasive strategy were excluded from the study. The baseline characteristics recorded included: medical history, vital signs, laboratory results, electrocardiographic findings, cardiac biomarkers, left ventricular ejection fraction (LVEF), door to device time, and angiographic findings. All angiographic features and procedural outcomes were reviewed by two investigators (SK and CW). Discrepancies in interpretation were resolved by consensus. The degree of stenosis was quantitated by visual analysis and significant stenosis was considered present when the narrowing of the coronary artery was $>70 \%$. The final epicardial TIMI was graded as previously described by the TIMI (Thrombolysis in Myocardial Infarction) group. ${ }^{9} 10$ The study was performed in accordance with the Declaration of Helsinki and the institutional ethics committee approved the use of data for this study and analysis.

\section{Definitions}

STEMI was defined as: (1) angina chest pain lasting $>30$ min with ST elevation $\geq 1 \mathrm{~mm}$ in two consecutive precordial or limb leads; or (2) angina chest pain lasting $>30$ min with new left bundle branch block. Cardiogenic shock at admission was defined as: (1) systolic blood pressure $<90 \mathrm{~mm} \mathrm{Hg}$ for $30 \mathrm{~min}$ despite adequate fluid therapy; (2) requirement for vasopressor infusion or intra-aortic balloon pump to maintain systolic blood pressure $>90 \mathrm{~mm} \mathrm{Hg}$; and (3) signs of poor tissue perfusion. Left ventricular function was assessed by echocardiography and left ventricular systolic dysfunction was defined as LVEF $<40 \%$. Renal dysfunction was defined as creatinine concentration $>1.5 \mathrm{mg} / \mathrm{dL}$ at admission. Anaemia was defined as haemoglobin concentration $<10 \mathrm{~g} / \mathrm{dL}$. Death included all causes of in-hospital death.

\section{Statistics}

Continuous variables were presented as mean $\pm S D$, while categorical variables were described as frequencies and percentages. Differences between the patient groups for categorical variables were examined using the $\chi^{2}$ or Fisher exact test or the $z$ test. Differences in the continuous variables between groups were assessed using the Student's t-test, 
Mann-Whitney U test, or Wilcoxon rank sum test, where appropriate. A univariate logistic model was used to examine the relationship between all clinical meaningful variables and in-hospital mortality. After each variable was tested independently in a univariate regression model, those that achieved a value of $\mathrm{p}<0.25$ (and were clinically meaningful) were selected for testing in a multivariable logistic regression. ORs and 95\% CIs were used to illustrate the association between potential variables and in-hospital mortality. A two-sided $\mathrm{p}$ value $<0.05$ was considered statistically significant. Stata V.10.0 was used to perform all statistical analyses (Stata Corp, College Station, Texas, USA).

\section{RESULTS}

\section{Baseline characteristic}

Between January 2008 and December 2011, 541 patients presenting with STEMI underwent primary PCI within 12 hours were included in the study. Of these 541 patients, 63 (11.6\%) died during hospitalisation. Death was due to cardiac causes in 58 patients $(92 \%)$ and non-cardiac causes in five patients $(8 \%)$. Table 1 summarises the baseline characteristic of all the patients. The study population was predominately male (69\%), with an average age of 63 years, and about one-third were diabetic. The mean total cholesterol and high density lipoprotein cholesterol values at admission were $189 \mathrm{mg} / \mathrm{dL}$ and $38.9 \mathrm{mg} / \mathrm{dL}$, respectively. Cardiogenic shock at admission was recorded in 301 patients $(55.6 \%)$ and intra-aortic balloon pump was used in $39 \%$ of all patients. About $95 \%$ of the patients received dual antiplatelet (aspirin and clopidogrel) therapy and only $20 \%$ of the patients received $\beta$-blockers or ACE inhibitors during hospitalisation. The in-hospital mortality rate in patients with admission cardiogenic shock was $76 \%$. Compared with the patients in the survival group, patients in the non-survival group were older, more likely to be female, non-smokers, have an LVEF $<40 \%$, be in cardiogenic shock at admission, exhibit renal dysfunction at admission, be anaemic, and have higher concentrations of admission troponin and plasma glucose.

\section{Coronary angiographic features and procedural outcomes}

Table 2 shows the coronary angiographic features and procedural outcomes. The median door-to-device time was $65 \mathrm{~min}$ and $\sim 80 \%$ of the patients underwent PCI within 90 min after admission. Coronary angiography showed that $78 \%$ of the patients $(n=424)$ had multivessel disease and the most commonly treated lesions were in the left anterior descending artery. The right coronary artery was found to be the infarct-related artery in $44 \%$ of all patients. There was no difference in number of vessel disease and the treated lesion between the survival and non-survival group. Thrombus aspiration was performed in $55 \%$ of all patients, and intracoronary glycoprotein IIb/IIIa was administered in $50 \%$ of all patients. Coronary stenting was performed in $86 \%$ of all patients. About $22 \%$ of the patients received drug eluting stents. Most of the infarct-related arteries were restored after PCI and the final TIMI grade 2/3 flow was achieved in $96.5 \%$ of the patients. The overall incidence of the no reflow phenomenon was $2.2 \%$.

Table 1 Baseline characteristic of the study patients

\begin{tabular}{|c|c|c|c|c|}
\hline Variables & Overall $(n=541)$ & Survive $(n=478)$ & Death $(n=63)$ & p Value \\
\hline Age, years $($ mean $\pm S D)$ & $63.5 \pm 12.1$ & $62.6 \pm 12.0$ & $69.8 \pm 10.8$ & 0.001 \\
\hline Male (n, \%) & $375(69.3)$ & $344(71.9)$ & 31 (49.2) & 0.001 \\
\hline $\mathrm{BMI}, \mathrm{kg} / \mathrm{m}^{2}(\mathrm{mean} \pm \mathrm{SD})$ & $23.3 \pm 3.9$ & $23.4 \pm 3.8$ & $23.0 \pm 3.7$ & 0.482 \\
\hline Pulse rate $($ mean $\pm S D)$ & $80.8 \pm 22.9$ & $80.4 \pm 21.7$ & $84.3 \pm 30.6$ & 0.213 \\
\hline Diabetes mellitus ( $\mathrm{n}, \%)$ & $163(30.1)$ & $139(29.8)$ & $24(38.1)$ & 0.152 \\
\hline Hypertension (n, \%) & $198(36.6)$ & $171(35.7)$ & $27(42.8)$ & 0.290 \\
\hline Smoking (n, \%) & $192(35.4)$ & $181(37.8)$ & $11(17.4)$ & 0.001 \\
\hline Previous CAD (n, \%) & $42(7.7)$ & $35(7.3)$ & $7(11.1)$ & 0.340 \\
\hline \multicolumn{5}{|l|}{ Killip classification } \\
\hline Class I (n, \%) & $145(26.8)$ & $137(28.6)$ & $8(12.7)$ & 0.004 \\
\hline Class II (n, \%) & $80(14.8)$ & $73(15.2)$ & $7(17.1)$ & \\
\hline Class III (n, \%) & $15(2.7)$ & $15(3.14)$ & $0(0)$ & \\
\hline Class IV (n, \%) & $301(55.6)$ & $253(52.9)$ & $48(76.2)$ & \\
\hline Cardiogenic shock at admission (n, \%) & $301(55.6)$ & $253(52.9)$ & $48(76.1)$ & 0.001 \\
\hline Creatinine $>1.5 \mathrm{mg} / \mathrm{dL}(\mathrm{n}, \%)$ & $127(23.4)$ & $102(21.34)$ & $25(39.68)$ & 0.001 \\
\hline Haemoglobin <10 g/dL (n, \%) & $75(13.8)$ & $56(11.7)$ & $19(30.1)$ & 0.001 \\
\hline Admission troponin $\mathrm{T}, \mathrm{ng} / \mathrm{mL}($ mean $\pm \mathrm{SD})$ & $4.6 \pm 7.5$ & $4.3 \pm 6.9$ & $6.9 \pm 10.7$ & 0.011 \\
\hline Admission plasma glucose, $\mathrm{mg} / \mathrm{mL}(\mathrm{mean} \pm \mathrm{SD})$ & $199.5 \pm 111.3$ & $191.6 \pm 104.7$ & $256.7 \pm 138.8$ & 0.001 \\
\hline Total cholesterol, $\mathrm{mg} / \mathrm{mL}(\mathrm{mean} \pm \mathrm{SD})$ & $189.8 \pm 75.0$ & $191.3 \pm 73.7$ & $172.4 \pm 89.5$ & 0.279 \\
\hline LVEF $<40 \%(n, \%)$ & $123(22.7)$ & $99(20.7)$ & $24(38.0)$ & 0.001 \\
\hline Median door-to-device time (min) & 65 & 65 & 60 & 0.734 \\
\hline Door-to-device time $>90 \mathrm{~min}$ & $119(22.0)$ & $108(22.5)$ & $11(9.2)$ & 0.331 \\
\hline Length of hospital stay (day) $($ mean $\pm S D)$ & $5.2(5.9)$ & $5.5(6.1)$ & $2.7(1.8)$ & 0.001 \\
\hline \multicolumn{5}{|l|}{ In-hospital medication } \\
\hline ASA $(n, \%)$ & $522.0(96.5)$ & $464.0(97.0)$ & $58.0(92.0)$ & 0.617 \\
\hline Clopidogrel $(n, \%)$ & $513.0(94.8)$ & $455.0(95.1)$ & $58.0(92.0)$ & 0.237 \\
\hline$\beta$-blocker (n, \%) & $123.0(22.7)$ & $122.0(25.8)$ & $1.0(1.6)$ & 0.001 \\
\hline ACEI $(n, \%)$ & $101.0(18.6)$ & $99.0(18.2)$ & $2.0(3.4)$ & 0.001 \\
\hline
\end{tabular}

ACEI, ACE inhibitor; ASA, acetylsalicylic acid (aspirin); BMI, body mass index; CAD, coronary artery disease; LVEF, left ventricular ejection fraction. 
Table 2 Angiographic and procedural characteristics

\begin{tabular}{|c|c|c|c|c|}
\hline Variables & Overall $(n=541)$ & Survive $(n=478)$ & Death $(n=63)$ & $\mathrm{p}$ Value \\
\hline Single vessel disease $(n, \%)$ & $117(21.6)$ & $97(20.2)$ & $20(31.7)$ & 0.262 \\
\hline Double vessel disease $(n, \%)$ & $167(30.8)$ & $151(31.5)$ & $16(25.4)$ & 0.611 \\
\hline Triple vessel disease $(\mathrm{n}, \%)$ & $257(47.5)$ & $230(48.1)$ & $27(42.8)$ & 0.605 \\
\hline Left main disease $(n, \%)$ & $54(9.9)$ & $46(9.6)$ & $8(12.7)$ & 0.444 \\
\hline Infarct related vessel=LAD ( $n, \%)$ & $266(49.1)$ & $236(49.3)$ & $30(47.6)$ & 0.856 \\
\hline Infarct related vessel=RCA $(\mathrm{n}, \%)$ & $241(44.5)$ & $213(44.5)$ & $28(44.4)$ & 0.990 \\
\hline Reference vessel diameter, $\mathrm{mm}(\mathrm{mean} \pm \mathrm{SD})$ & $3.26 \pm 0.08$ & $3.29 \pm 0.09$ & $3.00 \pm 0.08$ & 0.277 \\
\hline Stented implanted $(n, \%)$ & $468.0(86.5)$ & $421.0(88.1)$ & $47.0(74.6)$ & 0.003 \\
\hline Number of stent $(m e a n \pm S D)$ & $1.33(0.6)$ & $1.34(0.6)$ & $1.23(0.5)$ & 0.259 \\
\hline DES $(n, \%)$ & $121.0(22.3)$ & $113.0(23.6)$ & $8.0(12.7)$ & 0.549 \\
\hline Use of thrombus aspiration (n, \%) & $302(55.8)$ & $271(56.6)$ & $31(49.2)$ & 0.261 \\
\hline Glycoprotein Ilb/Illa inhibitor administered (n, \%) & $267(49.3)$ & $239(50.0)$ & $28(44.4)$ & 0.407 \\
\hline Initial TIMI flow grade 0/1 (n, \%) & $336.0(62.1)$ & $293.0(61.3)$ & $43.0(68.3)$ & 0.248 \\
\hline Final TIMI flow grade $0 / 1(n, \%)$ & $19(3.5)$ & $9(1.8)$ & $10(15.8)$ & 0.293 \\
\hline
\end{tabular}

DES, drug eluting stent; LAD, left anterior descending artery; RCA, right coronary artery; TIMI, Thrombolysis in Myocardial Infarction.

\section{In-hospital outcomes}

Univariate and multivariate analysis

In the univariate analysis (table 3), in-hospital mortality was independently related to: age $>60$ years, female sex, LVEF $<40 \%$, cardiogenic shock at admission, renal dysfunction at admission, haemoglobin $<10 \mathrm{~g} / \mathrm{dL}$, admission troponin, admission plasma glucose, reference vessel diameter, stent implantation, and final TIMI grade 0/1. After adjustment for baseline variables (table 4) in-hospital mortality was related to: age $>60$ years (OR 2.98, 95\% CI 1.17 to 7.05 ), LVEF < 40\% (OR $2.53,95 \%$ CI 1.20 to 5.36 ), and final TIMI grade 0/1 (OR $20.55,95 \%$ CI 3.49 to 120.94 ).

Table 3 Univariate analysis

\begin{tabular}{|c|c|c|c|}
\hline Variables & OR & $95 \% \mathrm{Cl}$ & p Value \\
\hline Age $>60$ years & 3.47 & 1.81 to 6.68 & 0.001 \\
\hline Female sex & 2.63 & 1.49 to 4.64 & 0.001 \\
\hline BMI & 0.97 & 0.91 to 1.04 & 0.482 \\
\hline Pulse rate & 1.00 & 0.99 to 1.02 & 0.213 \\
\hline Diabetes mellitus & 1.49 & 0.82 to 2.64 & 0.152 \\
\hline Hypertension & 1.33 & 0.75 to 2.34 & 0.289 \\
\hline Smoking & 0.33 & 0.15 to 0.66 & 0.001 \\
\hline Previous CAD & 1.58 & 0.67 to 3.71 & 0.420 \\
\hline Cardiogenic shock at admission & 2.82 & 1.50 to 5.57 & 0.001 \\
\hline Creatinine $>1.5 \mathrm{mg} / \mathrm{dL}$ & 2.42 & 1.33 to 4.33 & 0.001 \\
\hline Haemoglobin $<10 \mathrm{~g} / \mathrm{dL}$ & 3.30 & 1.69 to 6.27 & 0.001 \\
\hline Admission troponin $\mathrm{T}$ & 1.03 & 1.00 to 1.06 & 0.015 \\
\hline Admission plasma glucose & 1.04 & 1.002 to 1.006 & 0.001 \\
\hline LVEF $<40 \%$ & 3.01 & 1.60 to 5.62 & 0.001 \\
\hline Door-to-device time $>90 \mathrm{~min}$ & 0.71 & 0.32 to 1.44 & 0.330 \\
\hline Triple vessel disease & 0.57 & 0.30 to 1.06 & 0.077 \\
\hline Infarct vessel $=\mathrm{LAD}$ & 0.93 & 0.55 to 1.56 & 0.790 \\
\hline Infarct vessel=RCA & 0.99 & 0.59 to 1.68 & 0.980 \\
\hline Reference vessel diameter & 0.59 & 0.36 to 0.96 & 0.035 \\
\hline Stented implanted & 0.39 & 0.21 to 0.80 & 0.001 \\
\hline Use of glycoprotein IIb/IIla inhibitor & 0.80 & 0.45 to 1.40 & 0.400 \\
\hline Use of thrombus aspiration & 1.13 & 0.65 to 1.98 & 0.651 \\
\hline Final TIMI flow grade $0 / 1$ & 9.83 & 3.39 to 28.48 & 0.001 \\
\hline
\end{tabular}

\section{DISCUSSION}

The present study found that: (1) age $>60$ years, LVEF $<40 \%$, and final TIMI flow are related to in-hospital mortality; and (2) in-hospital mortality is high in unselected patients with STEMI transferred to the tertiary heart centre.

Although previous studies identified risk factors for shortand long-term outcomes for patients with ST elevation, it is unknown whether these can be generalised to all patients in modern daily practice. The emergence of primary PCI has dramatically improved outcomes for patients with STEMI in the last decade. ${ }^{11} 12$ The improvement in vessel patency and the higher rate of TIMI flow, and the reduction of fatal haemorrhagic stroke, are the key reasons for the success of primary PCI. Moreover, in-hospital mortality has consistently declined over the last decade. Age and LVEF are the most consistent predictors of mortality in STEMI since the introduction of aspirin and thrombolytic therapy. ${ }^{13-15}$ Recent clinical trials have moreover combined the clinical and angiographic information for identifying the predictors of mortality in STEMI. Impaired LV systolic function remains a dominant predictor of cardiovascular events among STEMI patients undergoing PCI. ${ }^{7}$ 16-19

Mounting evidence demonstrates that about half of patients with STEMI have multivessel coronary artery disease. ${ }^{20}{ }^{21}$ The incidence of multivessel coronary artery disease in the

Table 4 Multivariate analysis

\begin{tabular}{lcll}
\hline Variables & OR & 95\% Cl & p Value \\
\hline Age $>60$ years & 2.98 & 1.17 to 7.05 & 0.01 \\
Female sex & 1.33 & 0.60 to 2.92 & 0.48 \\
Smoking & 0.69 & 0.27 to 1.81 & 0.46 \\
Cardiogenic shock at admission & 1.78 & 0.82 to 3.87 & 0.14 \\
Creatinine $>1.5 \mathrm{mg} / \mathrm{dL}$ & 0.81 & 0.37 to 1.81 & 0.62 \\
Haemoglobin $<10$ g/dL & 2.19 & 0.97 to 4.93 & 0.06 \\
Admission troponin T & 0.99 & 0.95 to 1.04 & 0.83 \\
Admission plasma glucose & 1.00 & 1.000 to 1.006 & 0.01 \\
LVEF <40\% & 2.53 & 1.20 to 5.36 & 0.02 \\
Triple vessel disease & 1.86 & 0.73 to 4.72 & 0.19 \\
Reference vessel diameter & 0.99 & 0.69 to 1.42 & 0.97 \\
Stented implanted & 0.85 & 0.31 to 2.37 & 0.77 \\
Final TIMl flow grade $0 / 1$ & 20.55 & 3.49 to 120.94 & 0.001 \\
\hline
\end{tabular}

LVEF, left ventricular ejection fraction; TIMI, Thrombolysis in Myocardial Infarction. 
present study is $\sim 78 \%$, which is relatively high compared to previous studies. ${ }^{21} 22$ Although several studies have reported that a greater degree of coronary artery disease is associated with a worse prognosis in these patients, multivessel coronary artery disease was not an independent risk factor of in-hospital mortality in the present study. This indicates that primary PCI of the infarct-related artery may be sufficient to reduce in-hospital mortality.

System delays to reperfusion are correlated with higher rates of mortality and morbidity. ${ }^{2}$ Door-to-device time is critical and key to preserving the jeopardised muscle. ${ }^{3} 23$ Although the majority of the patients $(78 \%)$ in the present study underwent primary PCI within 90 min or less, a significant number of patients $(22 \%)$ still had a door-to-device time $>90 \mathrm{~min}$. The causes of in-hospital delay may due to the patient preparation process, including the blood sample draw process, electrocardiographic and echocardiographic examination, and resuscitation therapy in the emergency room. The skip emergency room strategy may reduce the door-to-device time and probably improve patient outcomes.

A key to the success of primary PCI in patients with STEMI is patency of the infarct related artery after revascularisation therapy. ${ }^{4}$ TIMI grade has been accepted as the gold standard for evaluating patency of the epicardial artery. Most of the patients in the present study (96.5\%) achieved TIMI flow grade $2 / 3$ and the overall incidence of no-reflow phenomenon was $2.2 \%$. Thrombus aspiration was performed in $55 \%$ of the patients and intracoronary glycoprotein IIb/IIIa inhibitor was administered in $49 \%$ of them.

The in-hospital mortality (11.6\%) was higher than that reported in previous clinical trials. ${ }^{24} 25$ Shihara et al ${ }^{22}$ reported in-hospital and 1-year mortality rates of $7.1 \%$ and $10.9 \%$, respectively. The high mortality rate in the present study may be attributable to the high proportion of patients with cardiogenic shock transferred to our heart centre. The non-high risk and stable patients were probably treated at local hospitals and transferred for risk stratification after the event.

The definition of cardiogenic shock and the difference of the study population may contribute to the higher prevalence of cardiogenic shock in this study compared to previous studies. $^{26} 27$ The incidence of acute myocardial infarction complicated by cardiogenic shock in a single community hospital reported by Goldberg et $a l^{26}$ was $7.1 \%$ and the in-hospital mortality was $71.7 \%$. Awad et $a l^{27}$ reported that the incidence of cardiogenic shock in the Global Registry of Acute Coronary Events (GRACE) registry was $4.6 \%$ and the overall mortality rate was $59.4 \%$. The haemodynamic criteria for cardiogenic shock, including pulmonary capillary wedge pressure and cardiac index information, were not available in the present study; therefore, other possible causes of hypotension including hypovolaemic shock, right ventricular infarction, and hypotension secondary to bradycardia could not be absolutely excluded. However, in the present study, most of the patients with admission cardiogenic shock (70.4\%) required intra-aortic balloon pump therapy to support their haemodynamic status, and may represent the high-risk STEMI population who required intensive cardiac care and early coronary interventions.

Risk stratification in patients with STEMI is crucial for guiding therapy and managing patient risk. The risk score and multivariable model derived from clinical trials comprised highly selected populations but may not include all patients in daily clinical practice. ${ }^{28}$ Differences in treatment strategy and the availability of coronary intervention may result in different outcomes. A TIMI risk score for STEMI is clinically useful for triaging and managing fibrinolytic-eligible patients, but may not suitable for those undergoing a revascularisation procedure in PCI-capable hospitals. ${ }^{13}$ The CADILLAC risk score defined seven risk factors (age $>65$ years, Killip class $2 / 3$, LVEF $<40 \%$, anaemia, renal insufficiency, triple vessel disease, and TIMI flow grade) that accurately predict short- and long-term outcomes in patients with STEMI undergoing primary PCI. $^{17}$ The CADILLAC risk score was derived from two clinical trials that excluded patients with cardiogenic shock at presentation. The GRACE risk score-a multivariable logistic regression model derived from patients with non-ST elevation and ST elevation acute coronary syndrome enrolled in GRACE-has not been verified for patients with STEMI undergoing primary PCI. ${ }^{29}$ Sanguanwong et $\mathrm{al},{ }^{30}$ using data from the Thai Acute Coronary Syndrome Registry (TACSR), reported that age $\geq 75$ years, diabetes, shock, and cardiac arrhythmias were predictors of in-hospital mortality in patients with STEMI. The total mortality rate in TACSR was $17 \%$ and primary PCI was performed in 22.24\% of all patients. Recently, the ALPHA score defined five variables (age, heart rate, systolic blood pressure, access site, and anterior localisation of the infarction) that were associated with 30-day mortality in patients with STEMI treated with primary PCI. ${ }^{31}$ The ALPHA score could be calculated without the need for ventriculography and blood tests.

\section{Limitations}

There are several limitations of the present study that should be acknowledged. First, the retrospective observational study design is subject to bias. Second, single centre operator experience should be taken into account. Third, patients who underwent rescue PCI and a pharmaco-invasive approach-as recommended by the current guideline-were excluded. Finally, the diagnosis of admission cardiogenic shock might be overestimated in this present study due to the lack of haemodynamic criteria for cardiogenic shock.

\section{CONCLUSION}

This study demonstrates that age $>60$ years, LVEF $<40 \%$, and final TIMI grade $0 / 1$ are significant predictors of in-hospital

\section{Key messages}

\section{What is already known about this subject?}

Aging and left ventricular function are predictors of short- and long-term mortality in patients with ST elevation acute coronary syndrome in randomised controlled trials.

What does this study add?

The present study adds important information on very high-risk patients with ST elevation myocardial infarction (STEMI) undergoing primary percutaneous coronary intervention in a tertiary heart centre. The proportion of patients with cardiogenic shock is higher than those reported in previous studies. The present study demonstrated that age, left ventricular ejection fraction, and final TIMI flow were associated with in-hospital mortality.

What might this impact on clinical practice?

The results of this study support the use of intensive treatment in high-risk patients with STEMI to improve the clinical outcomes. 
mortality in unselected patients with STEMI undergoing primary PCI. Our findings highlight the importance of LV function assessment before primary PCI, and suggest that optimal restoration of myocardial reperfusion during the PCI procedure is essential for improving patient outcomes.

Contributors SK: initiated the study design. CW and BP: provided the statistical analysis. All authors contributed to refinement of the study protocol, data collection and approved the final manuscript.

Competing interests None declared.

Ethics approval Khon Kaen University Ethics Committee.

Provenance and peer review Not commissioned; externally peer reviewed.

Data sharing statement The raw data will be provided on request at: sonkia@kku.ac.th.

\section{REFERENCES}

1 Steg PG, James SK, Atar D, et al. ESC guidelines for the management of acute myocardial infarction in patients presenting with ST-segment elevation. Eur Heart J 2012;33:2569-619.

2 O'Gara PT, Kushner FG, Ascheim DD, et al. 2013 ACCF/AHA guideline for the management of ST-elevation myocardial infarction: executive summary: a report of the American College of Cardiology Foundation/American Heart Association Task Force on Practice Guidelines. J Am Coll Cardiol 2013;61:485-510.

3 Dalby $M$, Bouzamondo $A$, Lechat $P$, et al. Transfer for primary angioplasty versus immediate thrombolysis in acute myocardial infarction: a meta-analysis. Circulation 2003;108:1809-14.

4 Keeley EC, Boura JA, Grines CL. Primary angioplasty versus intravenous thrombolytic therapy for acute myocardial infarction: a quantitative review of 23 randomised trials. Lancet 2003:361:13-20.

5 Andersen HR, Nielsen TT, Rasmussen $\mathrm{K}$, et al. A comparison of coronary angioplasty with fibrinolytic therapy in acute myocardial infarction. N Engl J Med 2003;349:733-42.

6 Abu-Assi E, Ferreira-Gonzalez I, Ribera A, et al. Do GRACE (Global Registry of Acute Coronary events) risk scores still maintain their performance for predicting mortality in the era of contemporary management of acute coronary syndromes? Am Heart J 2010;160:826-34.e1-3.

7 Ariza-Sole A, Teruel L, di Marco A, et al. Prognostic impact of chronic total occlusion in a nonculprit artery in patients with acute myocardial infarction undergoing primary angioplasty. Rev Esp Cardiol (Engl Ed) 2014;67:359-66.

8 Brener SJ, Mehran R, Brodie BR, et al. Predictors and implications of coronary infarct artery patency at initial angiography in patients with acute myocardial infarction (from the CADILLAC and HORIZONS-AMI Trials). Am J Cardiol 2011;108:918-23.

9 The effects of tissue plasminogen activator, streptokinase, or both on coronary-artery patency, ventricular function, and survival after acute myocardial infarction. The GUSTO Angiographic Investigators. N Engl J Med 1993;329:1615-22.

10 The Thrombolysis in Myocardial Infarction (TIMI) trial. Phase I findings. TIMI Study Group. N Engl J Med 1985;312:932-6.

11 Sidney S, Rosamond WD, Howard VJ, et al. The 'heart disease and stroke statistics--2013 update' and the need for a national cardiovascular surveillance system. Circulation 2013;127:21-3.

12 McNamara RL, Chung SC, Jernberg T, et al. International comparisons of the management of patients with non-ST segment elevation acute myocardial infarction in the United Kingdom, Sweden, and the United States: The MINAP/NICOR,
SWEDEHEART/RIKS-HIA, and ACTION Registry-GWTG/NCDR registries. Int I Cardiol 2014:175:240-7.

13 Morrow DA, Antman EM, Charlesworth A, et al. TIMI risk score for ST-elevation myocardial infarction: a convenient, bedside, clinical score for risk assessment at presentation: an intravenous nPA for treatment of infarcting myocardium early II trial substudy. Circulation 2000;102:2031-7.

14 Eagle KA, Lim MJ, Dabbous OH, et al. A validated prediction model for all forms of acute coronary syndrome: estimating the risk of 6-month postdischarge death in an international registry. JAMA 2004;291:2727-33.

15 Dokainish H, Rajaram M, Prabhakaran D, et al. Incremental value of left ventricular systolic and diastolic function to determine outcome in patients with acute ST-segment elevation myocardial infarction: the echocardiographic substudy of the OASIS-6 trial. Echocardiography 2013;31:569-78.

16 Halkin A, Stone GW, Dixon SR, et al. Impact and determinants of left ventricular function in patients undergoing primary percutaneous coronary intervention in acute myocardial infarction. Am J Cardiol 2005;96:325-31.

17 Halkin A, Singh M, Nikolsky E, et al. Prediction of mortality after primary percutaneous coronary intervention for acute myocardial infarction: the CADILLAC risk score. J Am Coll Cardiol 2005:45:1397-405.

18 Sorajja P, Gersh BJ, Cox DA, et al. Impact of multivessel disease on reperfusion success and clinical outcomes in patients undergoing primary percutaneous coronary intervention for acute myocardial infarction. Euro Heart J 2007:28:1709-16.

19 Brkovic V, Dobric M, Beleslin B, et al. Additive prognostic value of the SYNTAX score over GRACE, TIMI, ZWOLLE, CADILLAC and PAMI risk scores in patients with acute ST-segment elevation myocardial infarction treated by primary percutaneous coronary intervention. Int J Cardiovasc Imaging 2013;29:1215-28.

20 Shiraishi J, Kohno Y, Sawada T, et al. Predictors of in-hospital outcome after primary percutaneous coronary intervention for recurrent myocardial infarction. Cir $J$ 2008;72:1225-9.

21 Sanz G, Castaner A, Betriu A, et al. Determinants of prognosis in survivors of myocardial infarction: a prospective clinical angiographic study. N Engl J Med 1982:306:1065-70.

22 Shihara M, Tsutsui $H$, Tsuchihashi M, et al. In-hospital and one-year outcomes for patients undergoing percutaneous coronary intervention for acute myocardial infarction. Am J Cardiol 2002;90:932-6.

23 Andersen HR, Nielsen TT, Vesterlund T, et al. Danish multicenter randomized study on fibrinolytic therapy versus acute coronary angioplasty in acute myocardial infarction: rationale and design of the DANish trial in Acute Myocardial Infarction-2 (DANAMI-2). Am Heart J 2003;146:234-41.

24 Garcia E, Elizaga J, Perez-Castellano N, et al. Primary angioplasty versus systemic thrombolysis in anterior myocardial infarction. J Am Coll Cardiol 1999;33:605-11.

25 Grines $\mathrm{CL}$, Browne KF, Marco J, et al. A comparison of immediate angioplasty with thrombolytic therapy for acute myocardial infarction. The Primary Angioplasty in Myocardial Infarction Study Group. N Engl J Med 1993;328:673-9.

26 Goldberg RJ, Samad NA, Yarzebski J, et al. Temporal trends in cardiogenic shock complicating acute myocardial infarction. N Engl J Med 1999;340:1162-8.

27 Awad HH, Anderson FA Jr, Gore JM, et al. Cardiogenic shock complicating acute coronary syndromes: insights from the Global Registry of Acute Coronary Events. Am Heart J 2012;163:963-71

28 Lee KL, Woodlief LH, Topol EJ, et al. Predictors of 30-day mortality in the era of reperfusion for acute myocardial infarction. Results from an international trial of 41,021 patients. GUSTO-I Investigators. Circulation 1995;91:1659-68.

29 Granger CB, Goldberg RJ, Dabbous 0, et al. Predictors of hospital mortality in the Global Registry of Acute Coronary Events. Arch Intern Med 2003;163:2345-53.

30 Sanguanwong S, Srimahachota S, Tungsubutra W, et al. Predictors of in-hospital mortality in Thai STEMI patients: results from TACSR. J Med Assoc Thai 2007;90 (Suppl 1):91-7.

31 Hizoh I, Gulyas Z, Szabo G, et al. A new risk model for STEMI treated with primary PCl: the ALPHA score. J Am Coll Cardiol 2013;61:E98. 